\title{
Laser Breaks Liquid into Uniform Droplets
}

\section{A laser beam hitting a column of liquid controls the droplet pinch-off at the bottom of the stream.}

\section{By Rachel Berkowitz}

$$
\text { I }
$$

$\mathrm{n}$ inkjet technology, a stream of liquid under the influence of an electric field breaks into a sequence of droplets.

Researchers have now shown that such a stream can be controlled with a laser beam, which allowed them to produce highly uniform droplets [1]. The technique could lead to improved control over droplet breakup, and it demonstrates new possibilities for using optical forces to manipulate liquid-air interfaces.

A narrow liquid stream emerging downward from a nozzle, like water from a tap, is inherently unstable. Surface tension amplifies random surface waves that begin at the top and cause the stream to break into droplets at the bottom. Ordinarily, the droplets have a range of sizes and shapes and are randomly spaced. However, a steady and uniform sequence of droplets can be produced by exciting the stream with an external periodic driving force, typically mechanical, acoustical, or electrical [2]. If the driving frequency matches the column's intrinsic vibration frequency, large ripples develop along the surface that lead to uniform droplets.

Weiwei Deng and his colleagues at the Southern University of Science and Technology in China have long been interested in light-matter interactions, inspired by physics Nobel Laureate Arthur Ashkin, inventor of a technique for moving particles with light known as optical tweezers. "Ashkin also showed that a pulsed laser can bend a liquid-air interface, which is absolutely fascinating to us," says Deng.

The team's experiment involved pumping a jet of ethanol downward through a nozzle that could vary the jet's diameter from 120 to 500 micrometers. They focused a green laser beam onto the jet at a location between the nozzle and the point where the stream broke up into droplets.

By varying the beam's position and brightness, the researchers found that they could cause the jet to transition from a random breakup to an ordered breakup in which the droplets had nearly uniform diameter and spacing. In the ordered mode, the jet region immediately below the nozzle was alternately illuminated and dark, indicating that the laser beam was periodically transmitted upstream through the jet at the same frequency with which droplets were breaking off.

The team saw the same behavior in computer simulations of the experiment. They learned that the surface waves at the point where the laser hits the stream serve as an oscillating mirror that periodically reflects the laser beam upstream, and the liquid jet acts as an optical fiber. The researchers found that the main effect of the laser comes at the top of the jet, where the light concentrates in the meniscus region. The tiny perturbations of the surface produced by the light in this region are amplified lower down the jet and lead to the uniform droplet pinch-off. With the right light intensity and positioning, the liquid automatically "locks in" to the natural frequency that produces the uniform droplets.

Jean-Pierre Delville, of the University of Bordeaux in France points out that the effects imposed by a laser on a liquid surface are very weak. The small radiation pressure typically used can only deform a liquid surface by tens of nanometers, with surface tension preventing any further deformation. So he's impressed by the control that the team demonstrated. "This idea is very clever, because not only is the breakup perfectly controlled but also the usual [random production process] of drops is suppressed." 

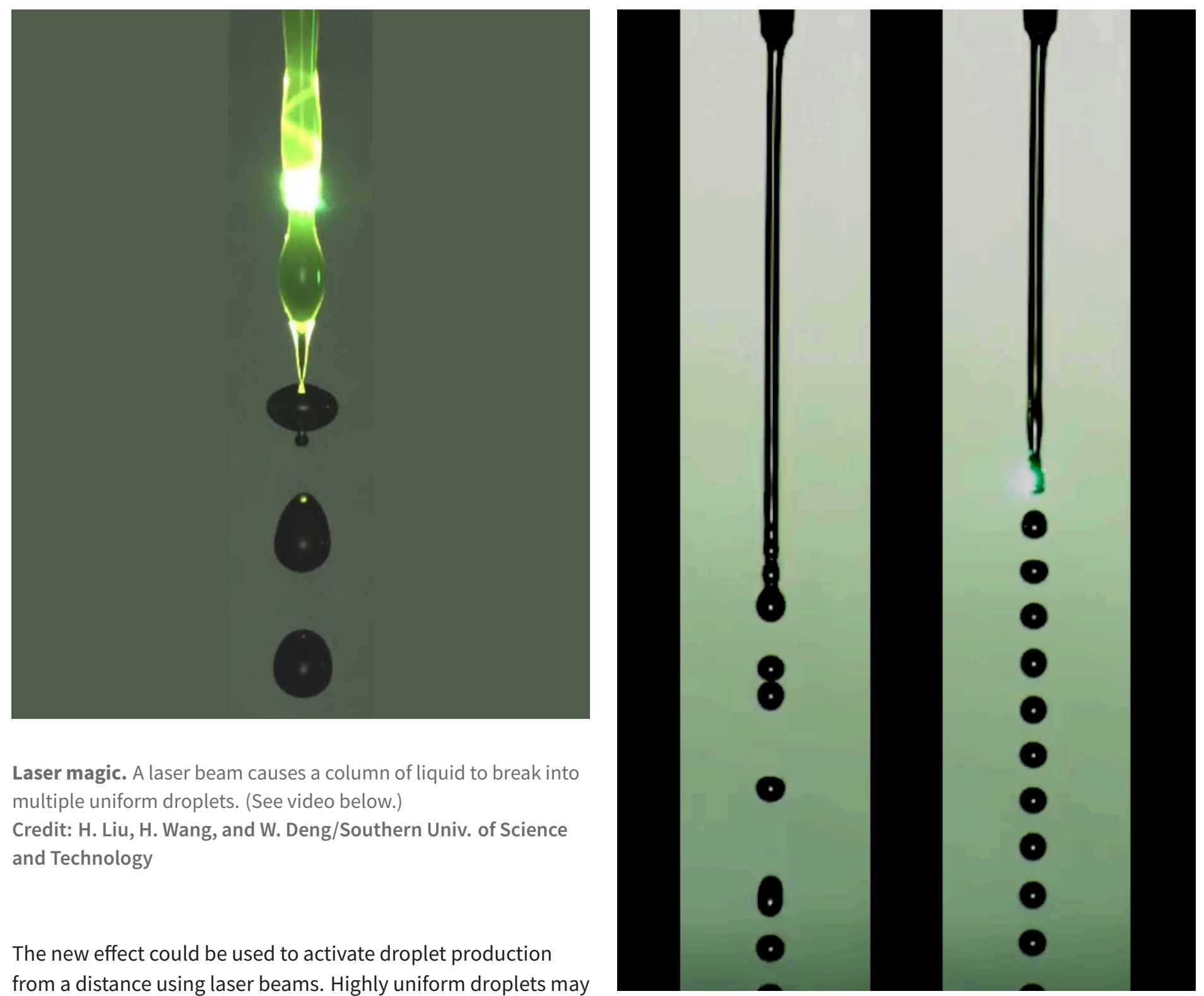

Laser magic. A laser beam causes a column of liquid to break into multiple uniform droplets. (See video below.)

Credit: H. Liu, H. Wang, and W. Deng/Southern Univ. of Science and Technology

The new effect could be used to activate droplet production from a distance using laser beams. Highly uniform droplets may be used in inkjet printing and in producing droplet size standards for calibrating aerosol instruments. But, says Deng, "we don't know exactly how to do that yet. It is the beauty of the physics that ultimately motivates us."

Rachel Berkowitz is a Corresponding Editor for Physics based in Vancouver, Canada.

\section{REFERENCES}

1. H. Liu et al., "Optofluidic resonance of a transparent liquid jet excited by a continuous wave laser," Phys. Rev. Lett. 127, 244502 (2021). 
2. W. Yang et al., "Crossover of varicose and whipping instabilities in electrified microjets," Phys. Rev. Lett. 112, 054501 (2014). 